AperTO - Archivio Istituzionale Open Access dell'Università di Torino

\title{
A Multidimensional Framework for the Representation of Ontologies in Adaptive Hypermedia Systems
}

\section{This is the author's manuscript}

Original Citation:

Availability:

This version is available http://hdl.handle.net/2318/89731

since 2018-03-08T14:20:40Z

Publisher:

Springer

Published version:

DOI:10.1007/11558590_38

Terms of use:

Open Access

Anyone can freely access the full text of works made available as "Open Access". Works made available under a Creative Commons license can be used according to the terms and conditions of said license. Use of all other works requires consent of the right holder (author or publisher) if not exempted from copyright protection by the applicable law. 


\title{
A Multidimensional Framework for the Representation of Ontologies in Adaptive Hypermedia Systems
}

\author{
Francesca Carmagnola, Federica Cena, Cristina Gena, Ilaria Torre \\ Dipartimento di Informatica, Università di Torino \\ Corso Svizzera 185, Torino, Italy \\ \{carmagnola,cena,cgena,torre\}@di.unito.it
}

\begin{abstract}
This paper introduces a semantic framework for adaptive systems. The core is a multidimensional matrix whose different planes contain the ontological representation of different types of knowledge. On these planes we represent user features, her actions, context, device, domain, adaptation goals and methods. The intersection between planes allows us to represent and managing semantic rules for inferring new user features or defining adaptation strategies. We exploit OWL to represent taxonomic knowledge and SWRL for rules.
\end{abstract}

\section{Introduction}

The Semantic Web aims at representing information in the WWW in a way such that machines can use it for automation, integration and reuse of knowledge across applications. The advantage of such an approach can be particularly useful in the field of adaptive hypermedia systems. These systems typically reflect some features of the user in the user model and apply this model to adapt various aspects of the system (content, interface, navigation, etc) to the user [6].

Current adaptive systems may also take into account other features, besides the user model, such as the context of interaction, the device, etc...

Usually the corpus of the documents and services the system can adapt is already known at the design time and can be defined as a closed corpus of adaptation [8]. The application of Semantic Web technologies to adaptive systems and the use of shared ontologies and metadata to describe resources can contribute to extend the closed corpus to an open corpus of adaptation. Thus, external documents and resources, which are semantically annotated, can be considered during the adaptation to the users. Furthermore, representing the user model with a semantic formalism and shared ontologies can be the base for building a user model server: a server that enables the reuse of user models, user modeling knowledge, and adaptation strategies across applications [12].

1 We are very grateful to Luca Console for having helped us during the development of the project and for his fruitful suggestions 
Different adaptive systems can query the same user model server, be provided with the user model and share the common knowledge.

This paper describes an ontology-based framework for adaptive hypermedia systems which aims at providing a methodological approach for the semantic definition of two types of knowledge:

(i) knowledge regarding what has to be adapted, which features (of the user, context, etc.) the system has to take into account to perform adaptation and how (adaptation methods and techniques);

(ii) knowledge regarding adaptation strategies and rules for inferring new knowledge.

Following the 'equation' ontology= (i) taxonomy + (ii), axioms, (see for example the RuleML Initiative [4]), we represent (i) the declarative descriptions of user models, domain knowledge, etc., with taxonomies expressed in a standard semantic markup language for the Semantic Web, $\mathrm{OWL}^{2}$, and (ii) the inference rules with SWRL ${ }^{3}$, a W3C proposal for a semantic rule language.

\section{Goals of the project and choices for semantic knowledge representation}

While many works in the user modeling and adaptation community exploit ontologies, in the form of taxonomies, to describe application domains and some recent ones adopt them to represent user models, devices features, context of interaction, etc. [8], [11], the semantic representation of reasoning strategies is still little addressed. In our project we use both taxonomies and reasoning strategies.

As far as taxonomies are concerned, we use them since they allow to represent and share conceptualizations of a certain knowledge domain [10] and contain a large set of pertinent concepts (entities, attributes) and the relations among them (IS_A, PART_OF, PORPUSE_OF, etc...).

The formalisms through which taxonomies may be expressed can be not XML-based, such as Kl_ONE [5] Lloom ${ }^{4}$, Flogic ${ }^{5}$; or XML-based, such as $\mathrm{XOL}^{6}$ (Ontology eXchange Language), $\mathrm{SHOE}^{7}$ (Simple HTML Ontology Extension), $\mathrm{OML}^{8}$ (Ontology Markup Language), OIL (Ontology Interchange Language) ${ }^{9}$, DAML (DARPA Agent Markup Language) $)^{10}$, DALM + OIL ${ }^{11}$, OWL (Web Ontology Language). Among

\footnotetext{
${ }^{2}$ http://www.w3.org/TR/owl-features/

${ }^{3}$ http://www.w3.org/Submission/SWRL/

${ }^{4}$ http://www.isi.edu/isd/LOOM/LOOM-HOME.html

${ }^{5}$ http://www.informatik.uni-freiburg.de/ dbis/Publications/95/flogic-jacm.html

${ }^{6}$ http://www.ai.sri.com/pkarp/xol/

${ }^{7}$ http://www.cs.umd.edu/projects/plus/SHOE/

${ }^{8} \mathrm{http} / / /$ www.ontologos.org/OML/OML\%200.3.htm

${ }^{9}$ http://www.ontoknowledge.org/oil/

${ }^{10} \mathrm{http}: / /$ www.daml.org/

${ }^{11}$ http://www.w3.org/TR/daml+oil-reference
} 
them OIL, DAML, OWL are compatible with web standard languages (RDF, RDF Schema) and give a support to reasoning strategies.

Regarding the representation language, we opted for OWL for two main reasons:

- it is the new standard ontology language of the Semantic Web, defined by W3C, and developed as revision of the previous DAML+OIL ${ }^{12}$;

- having a set of powerful primitives, mostly derived from description logic, it provides more expressive power than RDF and RDF schema.

What lacks in taxonomies is a set of reasoning mechanisms (which might be expressed by means of rules) to make inferences, and to extract useful information.

Thus, rule systems require taxonomies in order to have a shared definition of the concepts and relations mentioned in the rules, and taxonomies require a rule system to derive/use further information that cannot be captured by them. Rules allow also to add expressiveness to the representation formalism, to reason on the instances, and they can be orthogonal to the description logic taxonomies are typically based on.

Moreover, an ontology based on taxonomies and rules can provide humans (and machines) with rational explanations of system behaviour, thus improving their trust on the system. In the specific case of the Semantic Web, this is a relevant aspect for the so-called proof layer, which involves the "deductive process as well as the representation of proofs in Web Languages and proof validation"[2]. In this way, the proof presentation can be considered as a way for humans/machine to retrace the derivation of answers.

To achieve these goals, rules have to be expressed using semantic formalisms as well as taxonomies.

In our project, we exploit SWRL, a Semantic Web Rule Language combining OWL and RuleML ${ }^{13}$. In particular, SWRL is a combination of OWL Description Logic, OWL Lite and the Unary/Binary Datalog RuleML, and extends the set of OWL axioms to include Horn-like rules.

As described in the W3C proposal cited above, model theoretic semantics of SWRL is an extension of the semantics for OWL: it defines "bindings", which are extensions of OWL interpretations that map variables to elements of the domain: a rule is satisfied by an interpretation if every binding that satisfies the antecedent also satisfies the consequent. Therefore, OWL classes can be used as predicates in rules, and rules and ontology axioms can be freely mixed.

Like RuleML, SWRL allows interoperability with major rules systems (commercial and not): SQL, Prolog, CLIPS, JESS, etc...

Summarizing, a semantic representation of rules has different purposes, in particular:

- it enables knowledge sharing between software agents and human designers;

- it enables to compare and evaluate rules, detect incompatibilities, validate or eventually refuse them both in the design phase and in the exploitation phase;

\footnotetext{
${ }^{12}$ http://www.w3.org/TR/daml+oil-reference

${ }^{13} \mathrm{http} / / /$ www.ruleml.org/
} 
- in the field of adaptive systems, it allows to give explanations about the generation of inferences of new user features; the system adaptive behaviour and the strategies of adaptation

\section{Description of the framework}

The framework we propose aims at supporting the visual design, the semantic representation of knowledge bases and rules, and their implementation in adaptive hypermedia systems based on symbolic reasoning.

In addition to the above reasons, the choice of using a semantic formalism in order to define the framework arises from the evidence that user features are common to different applications and, if semantically described, they can be shared among them (consider for example the feature "user expertise": it is used by almost all adaptive systems). Defining these dimensions once for all represents an interesting opportunity in terms of reduced design costs and optimization of results. Moreover, the ontological representation of user, device, context and domain models also arises from the diffusion of this kind of taxonomies on the web (the last one in particular), and the possibility to link such taxonomies and integrating them with semantic web technologies and Web Services ${ }^{14}$.

For the definition of this semantic framework we developed a multidimensional matrix [14] composed of different planes. Each plane contains the ontological representation of a specific type of knowledge. In particular we have:

- user model taxonomy

- user actions taxonomy

- domain taxonomy

- device taxonomy

- context taxonomy

- adaptation goals taxonomy

- adaptation methods taxonomy

Regarding rules, the framework semantically represents and manages the typical and relevant rules in adaptive hypermedia systems:

- user modeling rules (which can be considered as derivation rules) that add knowledge about a user, inferring new user features from other features,

- adaptation rules (which can be considered as reaction rules) that define the strategies of adaptation, taking into account domain features, system adaptation goals, user features, context and the device in use.

Being a framework, the taxonomies on the planes have to be application independent and modular, so they can be reused among different domains and applications.

14 http://www.w3.org/TR/ws-arch/ 
In some planes we exploit and extend shared ontologies (in particular $\mathrm{CC} / \mathrm{PP}^{15}$ for the device, Ubisword ${ }^{16}$ for the user and the context features, the Open Directoy Project for the domain ${ }^{17}$ ), since they are easier to map, public available and better known.

Each taxonomy is defined at different levels: at the first level there is the definition of general concepts. For example, for the domain taxonomy, the first level includes macro domains such as: tourist domain, financial domain, e-learning domain, etc...; for the adaptation-goals taxonomy, the first level specifies general goals such as: inducing/pushing; informing, explaining, suggesting/recommending, guiding, assisting/helping [14], and so on for all the ontologies. At the following levels there are specialized concepts. For example, in the tourist domain, the next levels can include tourist categories (lodging, places, etc...), while in the adaptation-goals taxonomy they can include more specific goals such as explaining to support learning or to clarify, to teach new concepts or to correct mistakes, etc...

Thanks to this modular structure, the framework can be used by different applications, which can select a sub-part of the most generic taxonomy, in the considered planes, and instantiate only the concepts they are interested in.

The basic idea of the matrix is that user modeling and adaptation rules can be defined on the points of intersection between planes.

Given for example the leaf of the taxonomic tree "explaining $\rightarrow$ explaining to support learning $\rightarrow$ teaching new concepts", the idea is that the adaptation rule for reaching this goal (teaching new concepts) can be defined taking into account the knowledge domain, the user's current knowledge, her preferences and, possibly, her learning style (e.g. top-down vs. bottom-up), her current cognitive load, the current device (e.g. PDA, desktop pc) and context conditions (e.g. the noise level in the room). Finally, the definition of adaptation rules requires considering the set of available adaptation methods and techniques (such as hiding text, stretch text, audio annotations, direct guidance, etc.). Since all of these features are classes represented inside taxonomies in different planes, it can be perceived that the definition of the rule derives from the intersection of such planes in correspondence of the involved classes.

This methodology can be exploited to define all the rules addressed by the framework, clearly taking into account the appropriate planes.

User modeling rules. For this kind of derivation rules, which allow an adaptive system to infer new knowledge about the user, we consider:

- on the $\mathrm{X}_{1}$-plane, the taxonomy of the user's actions on the system (selection, bookmark, print, etc...);

- on the $\mathrm{X}_{2}$-plane, the taxonomy of the possible domain features (business, tourist, e-learning, shopping);

- on the $\mathrm{X}_{3}$-plane, the taxonomy of the user model (demographic features, psychographic features, cognitive features, preferences, interests, etc...);

\footnotetext{
15 http://www.w3.org/Mobile/CCPP/

${ }^{16} \mathrm{http}: / /$ www.u2m.org/

${ }^{17}$ http://dmoz.org
} 
From the intersection of dimensions on these planes we can define user-modeling rules in the form of:

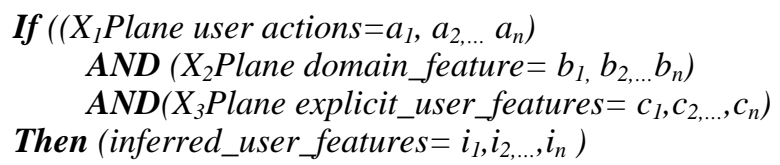

in which the Left Hand Side specifies classes or properties of classes that contribute to define the value of the inferred user's feature, which constitute the Right Hand Side.

For example:

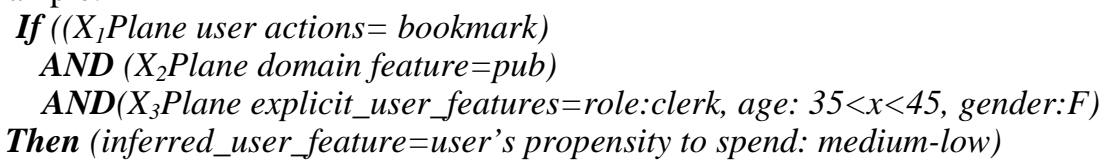

The matrix representation for this rule is showed in Fig. 1. This rule lets to infer the user's feature propensity to spend as a match between dimensions of each plane. In particular we assume that propensity to spend derives from the observation of variables such as user actions, domain features (as objects of users actions) and from specific user features (age, gender and role).

Giving that, the rule means that: if a user makes actions like bookmarking pages and the pages she has bookmarked regard pubs (in the tourist/town domain) and the user is a female, with an age between 35 and 45 years and she is a clerk, then we can infer that her propensity to spend may be medium-low.

Notice that, at this moment, we do not manage uncertainty or probability distributions of values, but we are working on defining a taxonomy of uncertainty factors and referencing it in SWRL.

Adaptation rules. As already explained, the above methodology can be used to define the adaptation rules as well, clearly changing the planes to take into account. Given that the aim of this matrix is to define the right adaptation techniques to reach a specific adaptation goal, given some available methods, a user, a device, a domain and a context, the taxonomies taken into account are the following:

- on the $\mathrm{Z}_{1}$-plane, we place the taxonomy of the adaptation goals (adaptation of content, interface, amount of information, detail level, etc.);

- on the $\mathrm{Z}_{2}$-plane we place the taxonomy of the adaptation methods (link removal, additional explanation, etc), defined following both Kobsa et. al. [13] and Brusilovsky [6] classifications.

- on the $\mathrm{Z}_{3}$-plane, we have the taxonomy of context conditions (e.g. time of the day, movement conditions,.)

- on the $\mathrm{Z}_{4}$-plane, the taxonomy of the user model integrated/updated with the user's dimensions inferred by the previous user modeling rules.

- on the $Z_{5}$-plane we place the taxonomy of devices that can be used by the user (PDA, PC, mobile phone, on-board system, DTT, etc.);

- on the $Z_{6}$-plane we place the taxonomy which describes the possible domain feature (business, tourist, e-learning, shopping). 


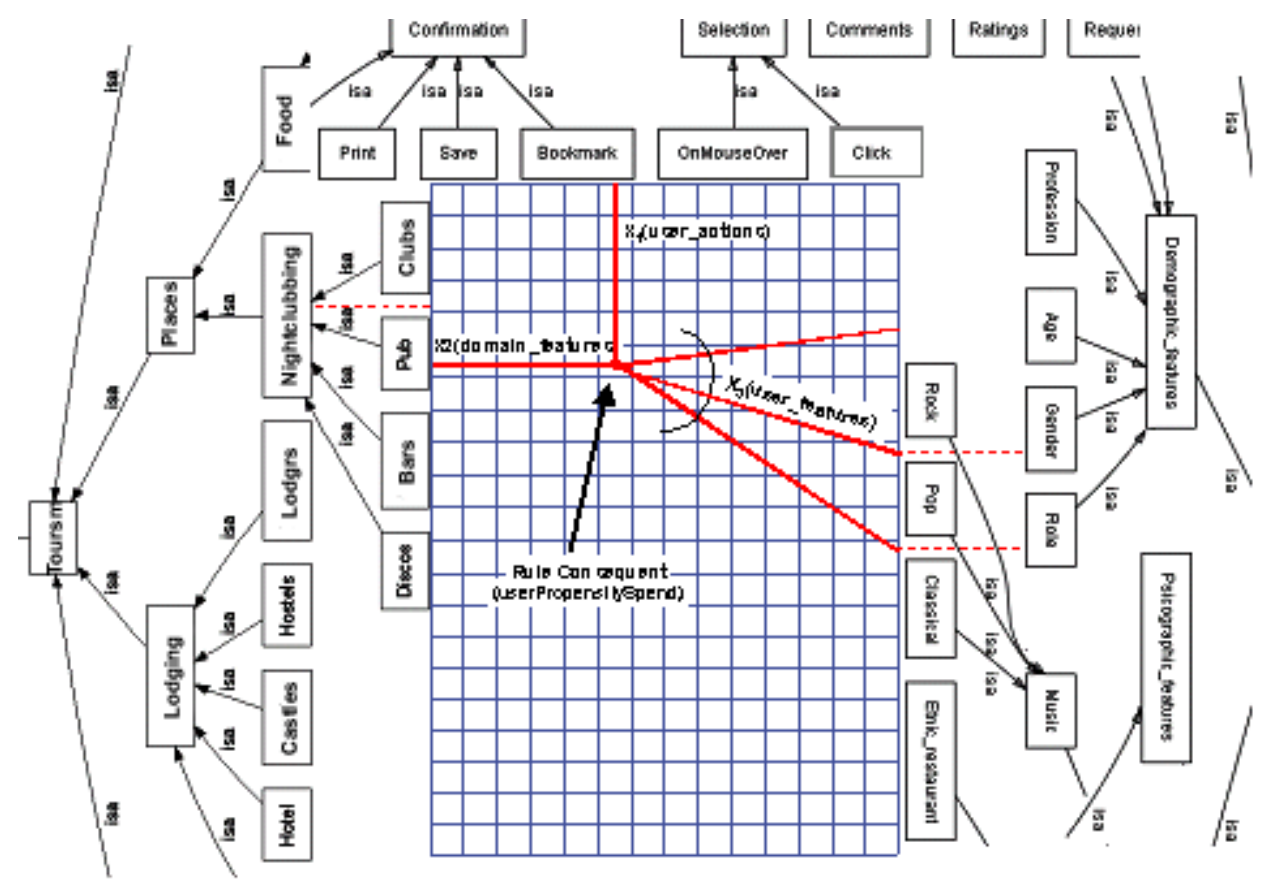

Fig. 1. A matrix for inferring user propensity to spend

The definition of the adaptation rule drives from the intersection of such planes:

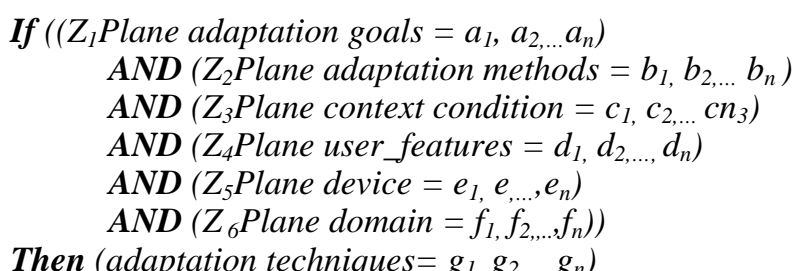

Then (adaptation techniques $=g_{1}, g_{2, \ldots,} g_{n}$ )

in which the Left Hand Side specifies the conditions to be satisfied and the Right Hand Side identifies the adaptation techniques for a correct method application.

For example:

If $\left(\left(Z_{1}\right.\right.$ Plane adaptation goal = emphasize an item $)$

AND ( $Z_{2}$ Plane adaptation methods = font enhancement, highlighting)

AND ( $Z_{3}$ Plane context condition=night, movement)

AND $\left(Z_{4}\right.$ Plane user_features=age: $\left.>65\right)$

$A N D\left(Z_{5}\right.$ Plane device $\left.=P D A\right)$

$\operatorname{AND}\left(Z_{5}\right.$ Plane domain $=\backslash$ any $)$ 


$$
\text { Then }(\text { adaptation techniques =font enhancement: }+3 \text { ) }
$$

According to this rule, having an elderly user in a nightly and mobile context, the system has to enlarge the font size in order to emphasise an item and to make it more readable.

As explained in section 2, taxonomies on the planes are written in OWL, while rules, at the intersection of planes, are written in SWRL.

A final consideration regards the explanation of how taxonomies and rules are employed to finally produce the User Model, Domain Model, Device Model, etc. of a specific application.

First of all we must underline that each component of the framework is ontologically represented as a class. Thus we have for example the User Model Class, the Domain Model Class, the Device Model Class, etc... each one characterized by a set of properties, that are the user features, the domain features, etc... so a specific user model represents an instance of the User Model Class.

Each property is mapped on the classes represented on the corresponding taxonomy. For example, the User Model property "Age" is mapped on the Class Age of the User Model Taxonomy. And the values of properties are the instances of the Classes in the corresponding taxonomy.

Inference and adaptation rules are written exactly taking into account the properties of the models, which are mapped on the taxonomies. The next section will provide an example.

\section{An example of application of the framework}

We are currently testing the proposed methodology with an application, UbiquiTo [1], we previously developed. This application is a multi-device adaptive guide that offers personalized tourist information. Therefore, the instantiation of the taxonomies on planes is restricted to the classes related to such features of the application.

For example, regarding the matrix for inferring user features, on the domain Plane, we consider the classes of the tourist domain (e.g., Lodging, Places, Arts, etc.). However, in Class Lodging, for instance, we do not instantiate the subclass "Castles", since UbiquiTo does not address it.

The same approach has been adopted for all the planes considered in the matrix. As said in section 3, some taxonomies are based on and extend public and shared ontologies (CC/PP for the device, Ubisword for the user and the context features, Open Directoy Project for the domain).

To support the development of the taxonomies and the translation in Owl, we use the free tool Protégé $3.0^{18}$. As it is a standard language we do not provide an example here. Instead, in the following we show an example of SWRL code for representing the above defined rule (see Fig.1), which derives user's "propensity_to_spend" starting from other user's features and user's actions on a specific domain.

// Definition of the taxonomies involved in the rule

${ }^{18}$ http://protege.stanford.edu/ 
$<$ swrl:Variable rdf:ID="user"/>

$<$ swrl:Variable rdf:ID="actions"/>

$<$ swrl:Variable rdf:ID="domain"/

$<$ ruleml:Imp $>$

\section{//Begin of the antecedent}

\section{// Definition of the URL of taxonomies}

$<$ ruleml:body rdf:parseType="Collection" $>$

$<$ swrl:classAtom $>$

$<$ swrl:classPredicate rdf:resource="URLOntology User"/>

$<$ swrl:argument1 rdf:resource="\#user"/ $>$

$<$ swrl:classAtom $>$

$<$ swrl:classAtom $>$

$<$ swrl:classPredicate rdf:resource="\& URLOntology Action "/>

$<$ swrl:argument1 rdf:resource="\#actions"/>

$</$ swrl:classAtom $>$

$<$ swrl:classAtom $>$

<swrl:classPredicate rdf:resource="\& URLOntology Domain"/>

$<$ swrl:argument1 rdf:resource="\#domain"/>

$</$ swrl:classAtom $>$

// Definition of the property "age" mapped on the user model taxomomy" $<$ swrlx: datavaluePropertyAtom swrlx:property="age">

$<$ ruleml:var $>$ user $</$ ruleml:var $>$

// Instatiation of the property "age” (value $35<x<45$ )

$<$ owlx:DataValue owlx:datatype="\&xsd;integer" $>35<x<45</$ owlx:DataValue $>$ $</$ swrlx:datavaluedPropertyAtom $>$

// Definition of the property "role" <swrlx:individualPropertyAtom swrlx:property="role"> $<$ ruleml:var $>$ user $</$ ruleml:var $>$

// Instatiation of the property "role" (value clerk) <owlx:Individual owlx:name="clerk"/> $<$ /swrlx:individualPropertyAtom>

// Definition of the property "gender" of the user model <swrlx:individualPropertyAtom swrlx:property="gender"> $<$ ruleml:var $>$ user $<$ /ruleml:var $>$

// Instatiation of the property "gender" of the user model (value female) <owlx:Individual owlx:name="female"/>

$<$ swrlx:individualPropertyAtom>

// Definition of the property "bookmark" of the user actions <swrlx:individualPropertyAtom swrlx:property="bookmark"> $<$ ruleml:var $>$ actions $</$ ruleml:var $>$ 
// Instatiation of the property "bookmark" of the user actions(value yes)

<owlx:Individual owlx:name="yes"/>

$<$ swrlx:individualPropertyAtom>

// Definition of the property "pub" of the domain

<swrlx:individualPropertyAtom swrlx:property="pub">

$<$ ruleml:var $>$ domain $<$ /ruleml:var $>$

// Instatiation of the property "pub" of the domain (value yes)

<owlx:Individual owlx:name="yes"/>

$<$ /swrlx:individualPropertyAtom>

//relation between an action (mapped on the user actions taxonomy) and a domain object (mapped on the domain taxonomy)

$<$ swrl:individualPropertyAtom>

$<$ swrl:propertyPredicate rdf:resource="Uri\#actions;objectOfAction"/>

$<$ swrl:argument1 rdf:resource="uri\#actions"/>

$<$ swrl:argument2 rdf:resource="uri\#domain"/>

$</$ swrl:individualPropertyAtom $>$

//End of antecedent

$<$ ruleml:body $>$

//Begin of the consequent

$<$ ruleml:head rdf:parseType="Collection">

$<$ swrl:individualPropertyAtom $>$

//Definition of new user's feature (propensity to spend) that emerges at the point of intersection between planes

$<$ swrl:propertyPredicate rdf:resource="\&URI;propensityToSpent"/>

$<$ ruleml:var $>$ user $</$ ruleml:var $>$

//Instatiation of the inferred feature "propensityToSpend" of the user model (value medium-low)

$<$ owlx:Individual owlx:name="medium low"/>

$<$ /swrlx:individualPropertyAtom $>$

$<$ swrl:argument1 rdf:resource="\#user"/>

$<$ swrl:argument2 rdf:resource="\#actions"/>

$<$ swrl:argument3 rdf:resource="\#domain"/>

$</$ swrl:individualPropertyAtom $>$

\section{Conclusion and Related Work}

In the recent years the User Modeling and Adaptive Hypermedia community has been approaching to Semantic Web technologies. Frasincar and Houben, for example, developed a methodology for the design of intelligent web information systems in the Web [9]. In this work, device capabilities are specified by means of CC/PP, while 
adaptation aspects, application domain, adaptivity conditions and update rules are expressed in RDFS. One of the most interesting aspects of their methodology is the design of the Application Model, which is concerned with the navigational aspects of the hypermedia presentation. They extended their Conceptual Model, expressed in RDFS, with navigational views, considered as slices of one ore more concepts from the Conceptual Model. Heckmann and Krueger [11] developed an XML-based markup language, UserML, and its corresponding ontology, UbisWorld, to communicate user models in a ubiquitous computing environment. Every UserML document can be divided into MetaData, UserModel, InferenceExplanations, ContextModel and Environment Model. The main aim of this representation is that different user modeling applications could use the same framework and keep their individual user model elements. Dolog et al. [8] developed an adaptive learning application using Semantic Web technologies. Learning resources are described by means of shared ontologies (Dublin Core and Learning Objects Metadata) with their RDF bindings and reasoning and adaptation are realized by using TRIPLE, a rulebased query language for the semantic web. Then, they also extended the adaptation capability of the systems to a global external context of semantically annotated resources, and they used TRIPLE to make ontology mapping, query relaxation, result filtering and finally to generate recommendations.

Respect to these works, the main contribution of our project is the definition of an ontological framework for managing rules and taxonomies in an integrated, semantic and visual way. In this framework we exploit the semantic formalism SWRL for the definition of reasoning capabilities and adaptation strategies integrating it with OWL, the standard language for the Semantic Web, which we exploit for the declaration of the knowledge base. Thus, through our framework, the development of an adaptive system may benefit from the availability of: i) shared ontologies regarding the user model, domain model, adaptation methods, etc. which the specific application can instantiate and extend, if necessary, ii) the matrix tool for representing, in a unified way, all the knowledge the system is based on, iii) standard and integrated languages for representing knowledge, iiii) implementation support, given by the possibility to convert OWL and SWRL to the syntax of rule engines such as CLIPS and Jess.

As regards future work, we are going to apply this methodology to other adaptive applications (e.g., [3], [7]) in order to evaluate if our approach is useful in different application domains and successful with different adaptation techniques. Regarding the extension of the framework, we are developing rules to integrate XSLT transformation in our resource and generate different kind of interfaces directly from our model. Moreover we are working to manage uncertainty defining a taxonomy of uncertainty factors and referencing it in SWRL. Finally, we are working on the extension of taxonomies on each plane.

\section{References}

1. Amendola I., Cena F., Console L., Crevola A., Gena C., Goy A., Modeo S., Perrero M., Torre I., Toso A.: UbiquiTO: A Multi-device Adaptive Guide. Proc. of Mobile HCI 2004, Lecture Notes in Computer Science, 3160 (2004) 409-414 
2. Antoniou, G., Van Harmelen F.: A Semantic Web Primer. The MIT Press, Cambridge, MA, (2004)

3. Ardissono L, Gena C., Torasso P., Bellifemine F., Chiaretto A., Difino A., Negro A. : Generation of personalized Electronic Program Guides. LNCS n. 2829. AI*IA 2003, Springer Verlag, (2003) 474-486

4. Boley, H., S. Tabet, and G. Wagner.: Design Rationale of RuleML: A Markup Language for Semantic Web Rules. Proc.of the First Semantic Web Working Symposium, SWWS'01, Stanford, CA, (2001) 381-402

5. Brachman R.J., Schmolze J.: An Overview of the KL-ONE Knowledge Representation System. Cognitive Sci 9(2) (1985)

6. Brusilovsky P.L.: Methods and Techniques of Adaptive Hypermedia. In User Modeling and User-Adapted Interaction 6 (1996) 87-129

7. Cena, F., Torre, I.: Increasing Performances and Personalization in the Interaction with a Call Center System. Proc. of the 8th ACM International Conference on Intelligent User Interfaces, Funchal, Madeira, Portugal, (2004), 226-228

8. Dolog, P., Henze, N., Nejdl, W., Sintek, M.: The Personal Reader: Personalizing and Enriching Learning Resources using Semantic Web Technologies. Proc. of the AH 2004, Eindhoven, The Netherlands, LNCS, Springer Verlag (2004) 85-94

9. Frasincar F., Houben G.: Hypermedia Presentation Adaptation on the Semantic Web. Proc. of the AH 2002, LNCS, Springer Verlag (2002) 85-94

10. Gruber R.: Toward Principles for the Design of Ontologies Used for Knowledge Sharing. Stanford Knowledge Systems Laboratory Technical Report KSL (1993) 9304

11. Heckmann D. and Krueger A.: A User Modeling Markup Language (UserML) for Ubiquitous Computing, in LNCS 2702, Springer Verlag (2003), 393 - 397

12. Kay, J., Kummerfeld, R., Lauder P.: Personis: A Server for User Models. Proc. of the AH, LNCS, Springer Verlag (2002) 203-212

13. Kobsa, A., Koenemann, J., Pohl, W.: Personalized Hypermedia Presentation Techniques for Improving Online Customer Relationships. The Knowledge Engineering Review 16(2) (2001) 111-155

14. Torre I.: Goals, tasks and Application domains as the guidelines for defining a framework for User modeling. In User Modeling 2001, LNCS, Springer Verlag (2001) 260-262 